\title{
Optimization of Recording of Thermal-Wave Images in BDM Method
}

\author{
Mateusz Kosikowski • Zbigniew Suszyński
}

Received: 8 April 2010 / Accepted: 1 February 2011 / Published online: 19 February 2011

(C) The Author(s) 2011. This article is published with open access at Springerlink.com

\begin{abstract}
The beam displacement modulation (BDM) method is very difficult to implement and has a lot of features and parameters which require optimization to improve the effectiveness of detection of thermal non-uniformities. The most important parameters that have to be taken under consideration during the optimization process are the width of the excitation source, the displacement speed of the excitation source, and the distance between the excitation and measuring spots. The width of the excitation source (e.g., diameter of laser beam) and speed of beam dislocation, in relation to the surface of the object, influence the spectra of excitation's energy. It allows examination and visualization of thermal properties of the object at varied depths. This article presents the general scheme of a thermal-wave microscope with the implemented BDM mode and describes the process of optimization for which the assignment is to find the microscope's settings that guarantee the highest temperature contrast of non-uniformities at the surface of the examined object.
\end{abstract}

Keywords Beam displacement modulation (BDM) - Measurement optimization · Temperature contrast · Thermal-wave microscopy

\section{Introduction}

The need of detection of non-uniformities in materials and electronic devices leads to the development of new research methods. This issue is particularly important from the viewpoint of the possibility of measuring thermal parameters of layered structures, as well as in examination of the quality of adhesion. One of the most popular

M. Kosikowski $(\varangle) \cdot$ Z. Suszyński

Division of Electronics and Computer Science, Koszalin University of Technology,

Sniadeckich Street 2, Koszalin 75453, Poland

e-mail: mateusz.kosikowski@tu.koszalin.pl 
methods in thermal microscopy is the so-called microphone-chamber method [1-3]. An advantage of this method is its high sensitivity of temperature measurement, but unquestionable disadvantages are large linear distortions and the need of placing the examined object in a photoacoustic chamber. Very often it causes a long time for the measurement [1]. Another well-known thermal-wave method is the one based on the so-called "flying-spot" or "beam displacement modulation" (BDM) generation of a thermal wave [4-7], which consists of the excitation of the object by a constant value of energy, but variable as to its place of dissipation. Information about thermal properties of the object is achieved by observation of the temperature response in the stimulation area or its vicinity. This method is already known for several years, but due to its analytical complexity and technical difficulties, has not found widespread applications. In this method the temperature response depends on parameters such as thermal properties of the object in the area of power excitation, the speed of movement of this area in relation to the detection device, and the size of the excited area and features of the excitation signal.

\section{Measurement Setup}

The technical solution proposed by authors consists of placing examined objects on the disk, which is rotating with a constant angular velocity (Fig. 1) [8]. An infrared detector and point of optical excitation are situated in such a way that the IR detector registers, with some delay in space and time, the changes of temperature caused by heating. The power of the excitation source is constant during the experiment. It was assumed that before the next sequential movement of the excitation beam over the same surface area, the local temperature disturbance is relaxed; therefore, the average temperature of the object is constant at the time of the experiment. Such an assumption is realistic in the case of structures characterized by a high thermal capacity.

The disturbance of the temperature field is recorded by the infrared detector located at a certain distance $\Delta x$ behind the place of stimulation, in relation to the direction

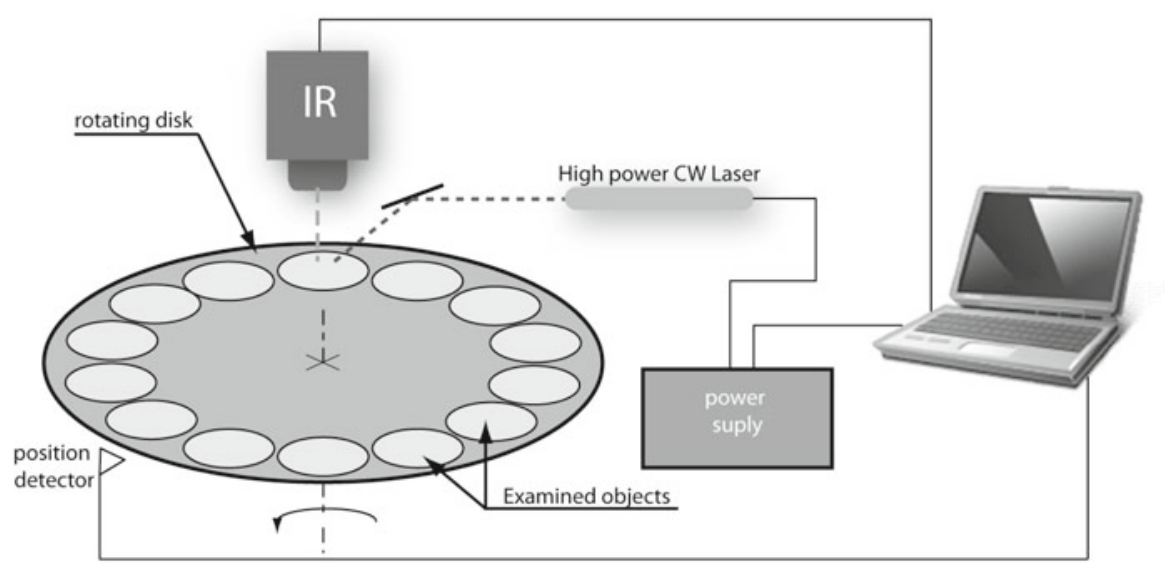

Fig. 1 Schematic of experimental setup for BDM method 


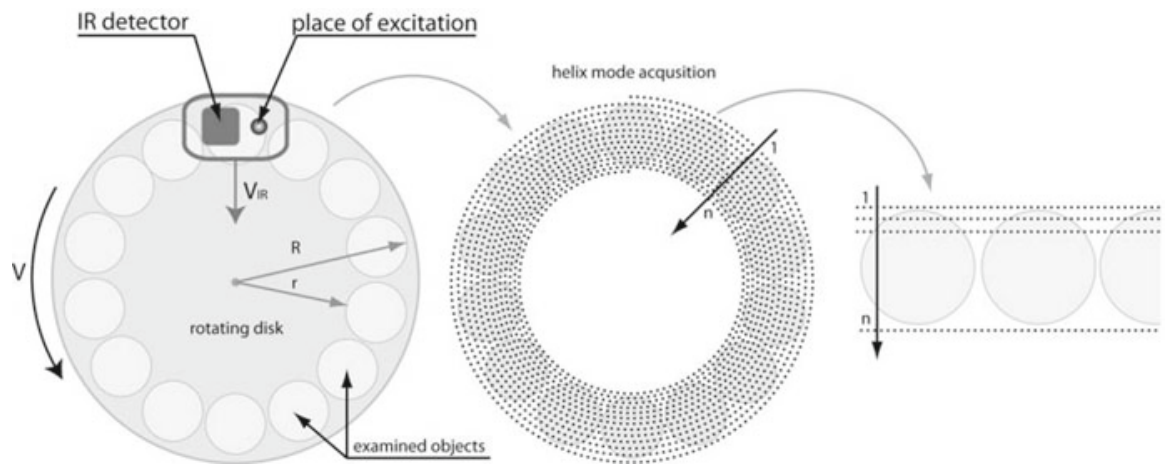

Fig. 2 Schematic of helix mode data acquisition

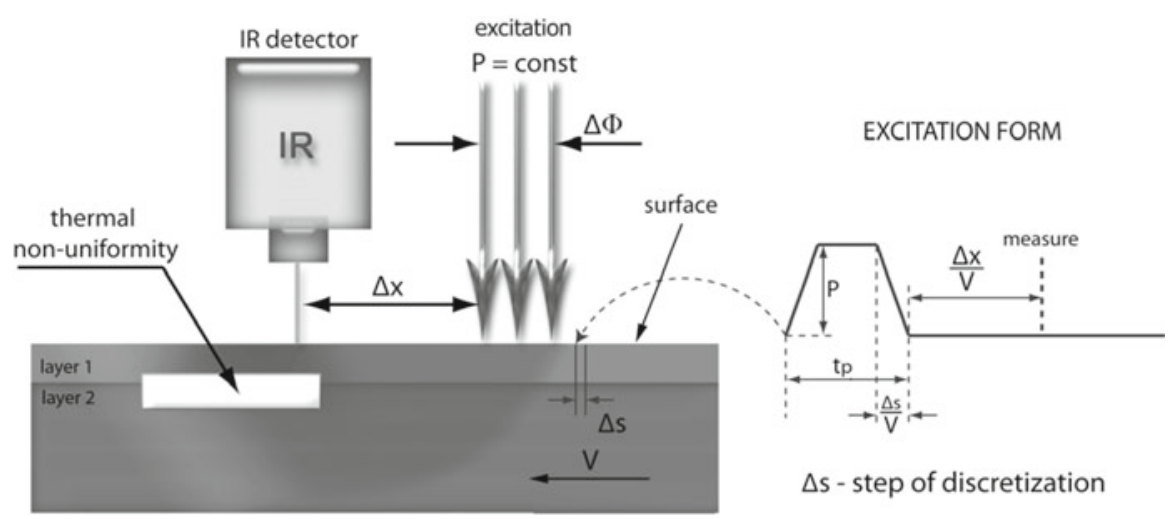

Fig. 3 Parameters and form of excitation

of the disk's rotation (Fig. 1). The thermal-wave image is created line by line for all objects at the same time. Examined objects are placed on the circle, as shown in Fig. 2. During a particular rotation of the disk, the IR detector and excitation source are shifted towards the center of the disk. Both the speed $V$ of the disk's rotation and the speed $V_{\text {IR }}$ of shifting the IR detector are constant during the experiment. Moreover, the speed $V_{\mathrm{IR}}$ should depend on the speed $V$, to ensure equal resolution of scanning in both directions. The full circle of the disk determines a transition to the new line of the created image. Authors assumed that the radius of the disk is big enough that the thermal-wave images can be presented as rectangular, as is shown in Fig. 2. The beginning of a new line is indicated by the synchronization device (Fig. 1). Such a configuration of the thermal-wave microscope allows one to measure the selected physical properties of all examined objects in a sufficiently short time. The recording time of the entire thermal-wave image is a function of the rotating speed of the disk and scanning resolution.

The speed $V$ and the distance $\Delta x$ define the time the single area $\Delta s$ of the object's surface (Fig. 3) is being excited and therefore it influences the frequency spectrum of the energetic excitation of the area $\Delta s$. In the described thermal-wave microscope, the 
$\mathrm{CW} \mathrm{CO}_{2}$ laser was used as a source of excitation, which supplies the object with a constant and continuous energy flux. However, from the viewpoint of a particular $\Delta s$ area of the surface, which is moving with speed $V$ in relation to the IR detector, the excitation has the shape of an isosceles trapezoid (Fig. 3). Assuming the $\Delta s$ is equal to the scanning resolution and is small enough, the excitation pulse takes a rectangular shape. In the special case, the $\Delta s$ parameter can be understood as a native resolution of the IR detector. The duration of excitation by the energy source, for the $\Delta s$ area of the object, is given by

$$
t_{\mathrm{p}}=\frac{\Delta \Phi}{V}
$$

In a thermal-wave flaw detector analyzed in this article, for a definite sampling resolution and diameter of the excitation beam, the speed of the moving object influences the frequency spectra of the excitation; therefore, it cannot be taken as arbitrary. The speed $V$ should depend on the thermal properties of materials, the width of the excitation pulse $\Delta \Phi$, as well as on the depth at which a thermal non-uniformity is located. A significant impact on the measurement is also the distance $\Delta x$ between the excitation area and the place of detection. Since described parameters $(V, \Delta \Phi, \Delta x)$ directly influence the quality of measurements, it is necessary to find their optimal values from the viewpoint of the sensitivity to the detected thermal non-uniformities.

\section{Optimization}

To optimize the recording conditions of the thermal-wave flaw detector in the BDM mode, the one-dimensional transmission line matrix (TLM) model of the layered structure was used [3] (Fig. 4). The temperature response $\Theta$ for harmonic excitation of such a system is defined by

$$
\Theta=Z_{\text {in }} P_{0} \mathrm{e}^{-j(\omega t)},
$$

where $Z_{\text {in }}$ is the input thermal impedance of the object in the frequency domain and $P_{0}$ is the power of the excitation signal at a given frequency.

In the case of multilayered structures, the input thermal impedance can be determined by

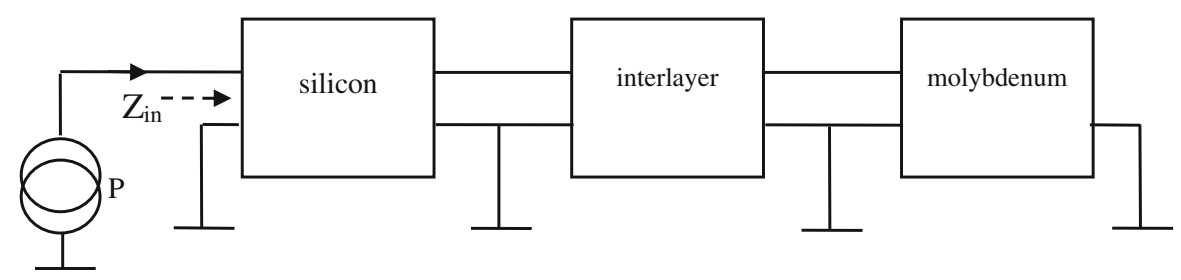

Fig. 4 Thermal model of layered structure 


$$
Z_{\text {in }}=z_{11}^{(1)}+\frac{\left(z_{12}^{(1)}\right)^{2}}{z_{11}^{(1)}+z_{11}^{(2)}-\frac{\left(z_{12}^{(2)}\right)^{2}}{z_{11}^{(2)}+z_{11}^{(3)}-\frac{\left(z_{12}^{(3)}\right)^{2}}{z_{11}^{(3)}+\cdots \quad}}}
$$

where $z_{11}^{(i)}=Z_{\mathrm{c}}^{i} \frac{\cosh \Gamma_{i}}{\sinh \Gamma_{i}}, z_{12}^{(i)}=Z_{\mathrm{c}}^{i} \frac{1}{\sinh \Gamma_{i}}, Z_{\mathrm{c}}^{i}=(1-i) \frac{1}{S \varepsilon_{i} \sqrt{2 \omega}}, \Gamma_{i}=(1+i) \sqrt{\frac{\omega}{2 \alpha_{i}}} l_{i}$, $Z_{0}$ is the thermal impedance to the environment (in $\mathrm{K} \cdot \mathrm{W}^{-1}$ ), $Z_{\mathrm{c}}^{i}$ is the characteristic impedance of the $i$ th layer (in $\mathrm{K} \cdot \mathrm{W}^{-1}$ ), $\alpha_{i}$ is the thermal diffusivity of the $i$ th layer $\left(\mathrm{m}^{2} \cdot \mathrm{s}^{-1}\right), \varepsilon_{i}$ is the thermal effusivity (in $\left.\mathrm{W} \cdot \mathrm{s}^{1 / 2} \cdot \mathrm{m}^{-2} \cdot \mathrm{K}^{-1}\right), S$ is the area of the excitation (in $\mathrm{m}^{2}$ ), and $l_{i}$ is the thickness of a particular layer (in $\mathrm{m}$ ).

The objective of the optimization process was to maximize the absolute temperature contrast $\Theta^{*}=\Theta_{\mathrm{d}}-\Theta$, defined as the difference between the temperature $\Theta_{\mathrm{d}}$ at the surface of the object at the site of a defect in relation to the temperature $\Theta$ of an area without a defect. The absolute temperature contrast, just the opposite to the relative temperature contrast defined in [4], gives the possibility to compare the temperature rise to the level of noises. This is very important for the BDM method because the measured temperature has a wide frequency spectrum, and therefore, the total power of the noise is relatively high in comparison with methods using harmonic excitation and lock-in signal detection.

The thermal contrast was calculated for the silicon-delamination-molybdenum structure in relation to the silicon-molybdenum one. The values of the thermal properties for each layer, which has been used for calculations, are presented in Table 1. It was assumed that the interlayer has the physical properties of air, so it is dissected. For the area of the object without a defect, such a layer was not included into the model. In the case of high-power thyristor structures, which were analyzed, possible delaminations are located at an exact depth. This is why it is not necessary to take under consideration more cases of different defects. Therefore, the goal of the optimization process was to find the maximum temperature contrast of the specified defected area in relation to the uniform area of the same object with proper adhesion. Since the physical properties of the object's layers are known, parameters that have to be optimized are $\Delta x, \Phi$, and $V$.

It should be noted that both the speed $V$ and distance $\Delta x$ affect the time delay between excitation and registration. In addition, the same speed $V$ and diameter $\Delta \Phi$ of the excitation beam define the time of excitation of the single area $\Delta s$ as is described by Eq. 1 . This is why the independent variables of the mathematical model should be $\Delta x V^{-1}$ and $V \Delta \Phi^{-1}$. The first one describes the time between excitation at a particular spot of the object and measuring in the same place. The second variable represents the main harmonic frequency which forms the rectangular shape of the excitation pulse. A lower frequency means the observation of thermal non-uniformities in greater depth inside the object. 
Table 1 Values of the thermal parameters used in the model

\begin{tabular}{llll}
\hline Layer & $\begin{array}{l}\text { Thermal effusivity } \\
\left(\mathrm{W} \cdot \mathrm{s}^{-1 / 2} \cdot \mathrm{m}^{-2} \cdot \mathrm{K}^{-1}\right)\end{array}$ & $\begin{array}{l}\text { Thermal diffusivity } \\
\left(\mathrm{m}^{2} \cdot \mathrm{s}^{-1}\right)\end{array}$ & $\begin{array}{l}\text { Thickness } \\
(\mathrm{m})\end{array}$ \\
\hline Silicon & 12569 & $6.33 \times 10^{-5}$ & $440 \times 10^{-5}$ \\
Interlayer (delamination) & 6 & $2.14 \times 10^{-5}$ & $1 \times 10^{-6}$ \\
Molybdenum & 18812 & $5.38 \times 10^{-5}$ & $2 \times 10^{-3}$ \\
\hline
\end{tabular}

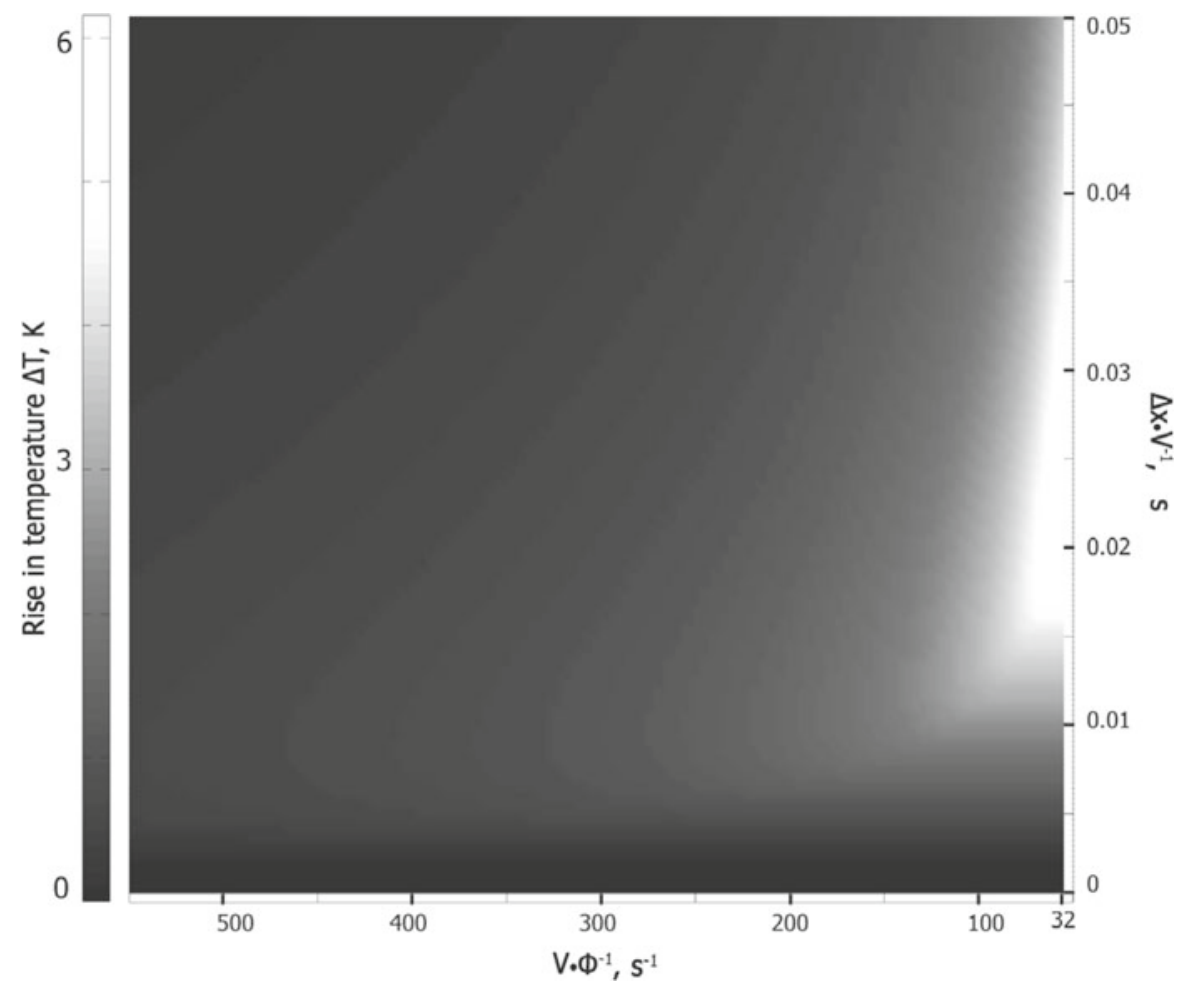

Fig. 5 Absolute temperature contrast of the object's thermal non-uniformity in relation to the area without defect for variant parameters of thermal-wave microscope

It was initially estimated that the technical parameters of the flaw detector, which should guarantee the highest temperature contrast during the measurements for the case of thyristor structures, are in the ranges $\Delta x V^{-1}=0.0015 \mathrm{~s}$ to $0.032 \mathrm{~s}$ and $V \Delta \Phi^{-1}=0 \mathrm{~s}^{-1}$ to $0.05 \mathrm{~s}^{-1}$. Moreover, it was assumed that the measurement resolution is so high that the excitation pulse has a rectangular shape. The power of excitation was assumed as $P=50 \mathrm{~W}$.

The results of model-based optimization are shown in Fig. 5. The temperature contrast is presented according to two independent model variables. The time delay $\Delta x V^{-1}$ for which the maximum temperature contrast for examined objects can be found is approximately $2 \mathrm{~ms}$ and decreases with increasing speed $V$ and decreasing diameter of excitation. Furthermore, the maximum temperature contrast can be found 
at lower frequencies, but, as was mentioned, delaminations in thyristor structures are located usually at the exact depth between silicon and molybdenum, so the maximum temperature contrast should not be found for the minimum frequency, as can be seen in Fig. 5. It would be in fact only for delaminations located very deeply. This nonconformity is conditioned by the one-dimensional model of heat flow. Such a model does not take into account the transverse section of the delamination and the diameter of the excitation beam. The two- or three-dimensional models make it possible to consider those parameters. Such an approach will be used by authors in future research. The advantage of a one-dimensional model is a fact of fast calculations. In spite of unrealistic values of the temperature contrast calculated with such a model for low frequencies, the result gives the possibility to approximately supply the optimal parameters required for examination of specified objects.

\section{Conclusions}

The main parameters that determine the sensitivity of detection of non-uniformities of the object are settings of the detection device. The optimization of those settings, and therefore the best adjustments of the flaw detector, may lead to maximizing the temperature contrast between the uniform and non-uniform areas of the object. The temperature contrast may be defined as an absolute or relative difference of temperature changes caused by a change of thermal properties of the object. For the BDM regime it is better to use the absolute difference of the temperature because it helps to estimate the signal-to-noise ratio. The goal for the optimization process was to achieve the maximum value of the temperature contrast for thermal parameters which should be observed. In the BDM method the temperature contrast strictly depends on the parameters $\Delta x, \Delta \Phi$, and $V$. Boundary conditions for the optimization function are limitations of construction parameters of the thermal-wave microscope. Because the function has only one maximum, the optimization can be limited to the graphic or gradient method. The BDM flaw detector which is described in this article is actually under construction, and the research presented in the paper gives the possibility to optimize the technical construction of the device. The experimental results achieved with the help of the described flaw detector will be presented in the future.

Acknowledgment This work was supported by the Ministry of Science and Higher Education in Poland, under Grant No. NN515247037.

Open Access This article is distributed under the terms of the Creative Commons Attribution Noncommercial License which permits any noncommercial use, distribution, and reproduction in any medium, provided the original author(s) and source are credited.

\section{References}

1. Z. Suszyński, IEEE Trans. Comp. Pack. Manufact. Technol. A 21, 434 (1998)

2. A. Rosencwaig, A. Gersho, J. Appl. Phys. 47, 64 (1976)

3. R.S. Quimby, W.M. Yen, J. Appl. Phys. 49, 2313 (1987)

4. R.S. Quimby, Appl. Phys. Lett. 39, 880 (1981)

5. Y.Q. Wang, P.K. Kuo, L.D. Favro, R.L. Thomas, Opt. Sci. 62, 24 (1990) 
6. Y. Wang, Ph.D. Thesis, Wayne State University, Detroit, MI (1993)

7. C. Gruss, F. Lepoutre, D. Balageas, Prog. Nat. Sci. 6, 173 (1996)

8. Z. Suszyński, M. Kosikowski, Elektronika 50(10), 99 (2009) 